\title{
Urinary lodine and Genetic Predisposition to Hashimoto's Thyroiditis in a Chinese Han Population: A Case-Control Study
}

\author{
Lu Li, ${ }^{1, *}$ Ying-Xia Ying, ${ }^{1, *}$ Jun Liang, ${ }^{2, *}$ Hou-Fa Geng, ${ }^{2}$ Qian-Yue Zhang, ${ }^{1}$ Chang-Run Zhang, ${ }^{1}$ \\ Fu-Xiang Chen, ${ }^{3}$ Yan Li, ${ }^{4}$ Yan Feng, ${ }^{4}$ Yan Wang, ${ }^{1,4}$ and Huai-Dong Song ${ }^{1}$
}

Background: We aimed to examine the association of urinary iodine concentration with Hashimoto's thyroiditis (HT) risk, and particularly, to investigate whether the HT-related genetic variations might modify the effects of urinary iodine on HT in the Chinese Han population.

Methods: We conducted a case-control study with 1723 Chinese (731 cases, 992 controls). The associations between urinary iodine concentration and HT risk were analyzed using logistic regression models. The effects of interactions between the genetic risk scores (GRSs) and urinary iodine on HT risk were assessed by including the respective interaction terms in the models. We also applied restricted cubic spline regression to estimate the possible nonlinear relationship. The multinomial logistic regression models were performed to determine the associations of urinary iodine with euthyroid-HT and hypothyroidism-HT.

Results: After controlling for potential confounders, the odds of HT increased with increasing quartiles of urinary iodine concentration: adjusted odds ratios (ORs) and 95\% confidence intervals [CIs] were 1.45 [1.061.99], 1.66 [1.17-2.34], and 2.07 [1.38-3.10] for the quartiles 2, 3, and 4, respectively, compared with the first quartile ( $p$ for trend $<0.001$ ). Multivariable restricted cubic spline regression analysis further demonstrated that there was a near-linear association between urinary iodine concentration and HT risk ( $p$-overall $<0.001$; $p$-nonlinear $=0.074)$. However, we did not find significant interactions between urinary iodine and GRSs on the risk of HT (all $p$ for interaction $>0.05$ ). Interestingly, we found that each increment of urinary iodine was associated with a more than twofold increase in the odds of hypothyroidism-HT (adjusted OR $=2.64$ $[\mathrm{CI}=1.73-4.05])$, but not with euthyroid-HT $(p>0.05)$.

Conclusions: Higher urinary iodine concentration was associated with increased risk of HT, and this association was near linear, indicating that increased urinary iodine has a continuous and graded impact on HT risk. Moreover, the iodine-HT association was not modified by genetic predisposition to HT. Interestingly, urinary iodine concentration was significantly associated with increased risk of hypothyroidism.

Keywords: Hashimoto's thyroiditis, urinary iodine, genetic risk score, interaction, single nucleotide polymorphisms, thyroid autoantibody

\section{Introduction}

$\mathbf{H}$ ASHIMOTO's THYROIDITIS (HT), an organ-specific autoimmune disorder, is characterized by intrathyroidal lymphocytic infiltration, progressive destruction of the thyroid follicles, and ultimately evolving into hypothyroidism (1). It is now widely considered as the most common autoimmune disease and the most frequent cause of hypothyroidism (2-6). Its incidence ranges from 27 to $448 / 100,000$ per year due to differences in study design and geographic areas (7). The rate of HT is increasing over the years (8), emphasizing that it is an important public health concern that needs more attention. Despite tremendous progress made in understanding of HT during the past decades, the exact mechanisms responsible for

\footnotetext{
${ }^{1}$ The Core Laboratory in Medical Center of Clinical Research, Department of Molecular Diagnostics and Endocrinology, Shanghai Ninth People's Hospital, State Key Laboratory of Medical Genomics, Shanghai Jiao Tong University (SJTU) School of Medicine, Shanghai, China.

${ }^{2}$ Department of Endocrinology, the Central Hospital of Xuzhou, Affiliated Hospital of Southeast University, Xuzhou, Jiangsu, China.

${ }^{3}$ Department of Clinical Immunology, Shanghai Ninth People's Hospital, Shanghai Jiao Tong University (SJTU) School of Medicine, Shanghai, China.

${ }^{4}$ School of Public Health, Shanghai Jiao Tong University (SJTU) School of Medicine, Shanghai, China.

*These authors contributed equally to this study.
} 
its progression still remain largely unclear (4). HT is a complex disease, involving the combination of both environmental and genetic factors $(1,4)$.

Of the environmental factors, the role of iodine has attracted increasing attention in recent years. Although enormous efforts have been put into research of the association of iodine intake with thyroid dysfunction, only a few studies have investigated its association with thyroid autoimmunity, and data are even more limited and inconsistent when focusing exclusively on clinically diagnosed HT (9-16). Interestingly, it has been suggested that iodine supplementation in previously iodinedeficient areas might lead to an increase in the prevalence of thyroid autoantibodies or accelerate the development of subclinical hypothyroidism to overt hypothyroidism (14-16). However, when administering $80 \mathrm{mg}$ potassium iodide to normal children for 3 months, Markou et al. found that none developed subclinical or overt hypothyroidism (17). These inconsistent findings might be attributed, at least partly, to the heterogeneity of the study population. In fact, previous epidemiological studies have shown that iodine supplementation increased the prevalence of hypothyroidism among individuals with autoimmune thyroiditis $(14,18,19)$. Supporting evidence was also provided by utilizing experimental animal models, where excessive iodine intake induced and aggravated autoimmune thyroiditis in a dose-dependent manner in NOD.H$2 \mathrm{~h} 4$ mice (20), a genetically predisposed model of autoimmune thyroiditis that closely resembles human disease (1), but not in SJL or C57BL/6 mice (21), mice strains without the genetic propensity for thyroiditis. Therefore, it is tempting to presume that iodine supplementation might trigger or exacerbate thyroid autoimmunity in the susceptible populations with either iodine deficiency or potential autoimmune thyroiditis. However, in humans, no published studies have so far focused on the interaction between the iodine intake and genetic predisposition to HT risk.

The considerable heritability of HT (about 75\%) instigated extensive efforts to unravel its genetic background (22), but so far, the majority of HT-susceptible genes were based on candidate gene studies with relatively small sample sizes (23-27), leaving potential susceptibility gene variants still poorly understood $(28,29)$. More recently, our group performed studies in the largest number of samples to date of Chinese Han ancestry (30) and identified or replicated 13 susceptibility loci for HT, providing valuable tools to examine the impact of gene-environment interaction on HT, especially in Chinese population. On the basis of this, in the current study, by constructing genetic risk scores (GRSs), which present the combined effect of 13 genetic variations, we conducted a case-control study to assess the association of urinary iodine as well as GRSs with HT risk and further to investigate whether the HT risk-related GRSs might modify the effects of urinary iodine on HT risk in a Chinese Han population aged 18 years or older. Finally, we also analyzed the association between urinary iodine and thyroid function status by stratifying HT patients according to whether the hypothyroidism was present or not.

\section{Materials and Methods}

\section{Study population}

A total of 1723 unrelated Chinese Han individuals aged 18 years or older, including 731 HT cases and 992 healthy controls, were recruited from April 2017 to February 2018 in Xuzhou, Jiangsu Province, China. Cases were recruited at Endocrinology and Metabolism Clinics of Xuzhou Central Hospital. HT was diagnosed based on the presence of diffuse swelling of the thyroid gland and the positivity for thyroid peroxidase antibody (TPOAb) (defined as TPOAb $>9.0$ $\mathrm{IU} / \mathrm{mL}$ ) and/or thyroglobulin antibody (TGAb) (defined as TGAb $>115.0 \mathrm{IU} / \mathrm{mL}$ ), together with typical HT appearance on thyroid ultrasound characterized as echo-structure with diffuse or patchy hypoechogenicity (5). The HT cases were further classified into HT with euthyroid (euthyroid-HT) and HT with hypothyroidism (hypothyroidism-HT) based on their thyroid function status. The euthyroid-HT group included patients whose thyrotropin $(\mathrm{TSH})$ (reference range, 0.34 $5.60 \mu \mathrm{IU} / \mathrm{mL}$ ) and free thyroxine (fT4) (reference range, 0.58 $1.64 \mathrm{ng} / \mathrm{dL}$ ) were within normal reference ranges and were not on L-thyroxine replacement therapy. The hypothyroidism-HT group included patients with subclinical hypothyroidism (defined as TSH $>5.60 \mu \mathrm{IU} / \mathrm{mL}$, fT4 within the normal range), or overt hypothyroidism (defined as TSH $>5.60 \mu \mathrm{IU} / \mathrm{mL}$, fT4< $0.58 \mathrm{ng} / \mathrm{dL}$ ), or on L-thyroxine replacement. The control subjects without any evidence of HT were recruited from individuals who visited outpatient clinics of the participating hospital for a healthy checkup. Medical history of the controls was examined, and those with a personal or family history of autoimmune diseases or thyroid diseases were excluded. There were no specific matching criteria for controls. Individuals were not eligible for enrollment if they had severe hepatic, cardiac, or kidney diseases, communication disorders, or had other thyroid disorders (such as Graves' disease). After a stringent quality control of genotyping, individuals with a missing rate more than $5 \%$ of genotyping data (i.e., those with missing information for any of the 13 single nucleotide polymorphisms [SNPs]) were removed.

The study protocol was approved by the Ethics Committee of Shanghai Ninth People's Hospital affiliated to Shanghai Jiao Tong University School of Medicine. All participants provided written informed consent before enrollment.

\section{Anthropometric and laboratory measurements}

Information on lifestyle factors, family history of any thyroid disease, medication history, current and past thyroid disease, and sociodemographics was recorded by trained personnel using a detailed questionnaire. Body height and weight were measured, and body mass index (BMI) was calculated by dividing the weight in kilograms with the square of the height in meters.

Serum samples were drawn after an 8-hour overnight fast. As described previously (31), high-density lipoprotein cholesterol (HDL-C), low-density lipoprotein cholesterol (LDL-C), triglycerides, and total cholesterol were determined by applying an enzymatic assay on Siemens ADVIA 2400 automatic analyzer. Both intra- and inter-assay coefficients of variation (CVs) were $<2.5 \%$ for HDL-C, $<10 \%$ for LDL-C, $<1.8 \%$ for triglycerides, and $<1.1 \%$ for total cholesterol. Serum free triiodothyronine (fT3) (reference range, 2.5-3.9 pg/mL), fT4 (reference range, $0.58-1.64 \mathrm{ng} / \mathrm{dL}$ ), TSH (reference range, $0.34-5.60 \mu \mathrm{IU} / \mathrm{mL}$ ), and TPOAb (reference range, $<9.0 \mathrm{IU} / \mathrm{mL}$ ) were tested using chemiluminescence immunoassay (UniCel DxI 800 Immunoassay System; Beckman Coulter). Serum TGAb (reference range, $<115.0 \mathrm{IU} / \mathrm{mL}$ ) 
was measured by an electrochemiluminescence immunoassay (Cobas 6000; Roche Diagnostics, Germany). The intra- and inter-assay CVs were 2.6-6.6\% and 1.3-8.0\% for fT3, 1.8$4.4 \%$ and $3.3-8.0 \%$ for fT4, $2.0-4.0 \%$ and $0.2-2.0 \%$ for TSH, $5.1-7.1 \%$ and $2.2-4.4 \%$ for TPOAb, $1.8-2.1 \%$ and $4.6-5.1 \%$ for TGAb, respectively.

\section{Assessments of urinary iodine and creatinine}

Spot urine samples were collected from all participants in the morning. Urinary iodine concentration was measured by the modified Sandell-Kolthoff reaction, which was a standard promulgated by the Ministry of Health of the People's Republic of China (WS/T 107-2006), as described previously $(31,32)$. The intra- and inter-assay CVs for urinary iodine concentration were $2-5 \%$ and $3-6 \%$ at $152 \mu \mathrm{g} / \mathrm{L}$, respectively. An enzymatic assay was applied to determine urinary creatinine concentration on Siemens ADVIA 2400 automatic analyzer, and both intra- and inter-assay CVs were $<3.2 \%$. With the advantages of the best technical feasibility, the lowest cost, and the absence of creatinine variability linked to $\mathrm{pH}$ condition, protein intake, muscle mass, sex, age, and ethnic background (33-35), the measurement of urinary iodine concentration $(\mu \mathrm{g} / \mathrm{L})$ from spot urine sample, recommended by the World Health Organization (WHO) (36) and also the most widely used currently (33), was mainly applied to the present study to estimate iodine status of the population.

All serum and urine samples were processed using the same instruments and kits in the central laboratory.

\section{Ultrasonography evaluation}

Thyroid ultrasonography was performed in all participants with 7-12 MHz linear transducer (Philips iE33 system; Philips Medical, Best, The Netherlands) by the certified ultrasonographers. An abnormal appearance of thyroid grand, charac- terized by diffuse areas of reduced echogenicity, was regarded as a criterion for the sonographic diagnosis of HT (5).

\section{SNP selection and genotyping}

We selected and genotyped 13 HT-associated SNPs that reached the Bonferroni corrected significance $p$ threshold calculated as 0.05 divided by the effective number of independent tests, based on our recent candidate-gene approach studies performed in Chinese Han population (30), with the largest number of samples to date. The detailed list of these SNPs is provided in Table 1. Genomic DNA was extracted from peripheral blood using commercial DNA extracting kit (QuickGene-DBL; KURABO Co., Ltd, Japan) on an automated instrument (QuickGene-610L; KURABO Co., Ltd). The genotyping of these SNPs was performed in Fluidigm EP1 platform with the 192.24 Dynamic Array integrated fluidic circuit (Fluidigm Corp., South San 129 Francisco, CA) using fast TaqMan assays (Applied Biosystems, Foster City, CA) in accordance with the manufacturer's instructions. Stringent quality control criteria, as detailed previously $(30,37)$, were used to select both individuals and genotyped SNPs for analysis. No linkage disequilibrium was found between the SNPs $\left(r^{2}<0.8\right)$, and the concordance rate was more than $99 \%$ based on 100 duplicates genotyping.

\section{Genetic risk scores}

We computed two polygenic risk scores, "unweightedGRS" and "weighted-GRS," based on the 13 SNPs selected in the current study. An unweighted-GRS was constructed for each participant by simply summing up the risk alleles across the 13 SNPs assuming an equal magnitude of effect at each locus, which seems to be more plausible when the genetic model is unknown. We also developed a weighted-GRS that utilized the allelic odds ratios (ORs) from our previous study with the largest sample size to date to account for the strength

Table 1. Single Nucleotide Polymorphisms That Compose the Genetic Risk Scores AND Weights Assigned to EACH Marker

\begin{tabular}{|c|c|c|c|c|c|c|c|c|c|c|}
\hline Chr. & SNP & $\begin{array}{l}\text { Risk } \\
\text { allele }\end{array}$ & $\begin{array}{l}\text { Case } \\
\text { RAF }\end{array}$ & $\begin{array}{c}\text { Control } \\
R A F\end{array}$ & $\begin{array}{l}\text { Base pair } \\
\text { location }\end{array}$ & $\mathrm{p}$ & $O R$ & Gene & Weight & Case/control \\
\hline 1 & rs 1265883 & $\mathrm{C}$ & 0.14 & 0.09 & 160464911 & $3.67 \times 10^{-16}$ & 1.59 & SLAMF6 & 0.4637340 & $2682 / 3892^{\mathrm{a}}$ \\
\hline 1 & rs7537605 & A & 0.35 & 0.30 & 108343087 & $5.49 \times 10^{-8}$ & 1.23 & VAV3 & 0.2086389 & $2864 / 3185^{\mathrm{b}}$ \\
\hline 2 & rs 1881145 & A & 0.63 & 0.59 & 12634278 & $2.00 \times 10^{-6}$ & 1.19 & TRIB2 & 0.1739533 & $2682 / 3892^{a}$ \\
\hline 2 & rs 1024161 & $\mathrm{~T}$ & 0.73 & 0.68 & 204721752 & $2.31 \times 10^{-11}$ & 1.31 & CTLA4 & 0.2700271 & $2682 / 3892^{a}$ \\
\hline 2 & rs11675434 & $\mathrm{T}$ & 0.33 & 0.30 & 1407815 & $5.02 \times 10^{-5}$ & 1.16 & $T P O$ & 0.1475576 & $2864 / 3185^{\mathrm{b}}$ \\
\hline 3 & rs 13093110 & $\mathrm{~T}$ & 0.63 & 0.60 & 188125120 & $5.89 \times 10^{-4}$ & 1.14 & $L P P$ & 0.1310283 & $2682 / 3892^{a}$ \\
\hline 4 & rs6832151 & $\mathrm{G}$ & 0.38 & 0.35 & 40303633 & $1.06 \times 10^{-4}$ & 1.16 & CHRNA9/RHOH & 0.1484200 & $2682 / 3892^{a}$ \\
\hline 6 & rs 1521 & $\mathrm{~T}$ & 0.84 & 0.78 & 31350704 & $2.81 \times 10^{-21}$ & 1.55 & $M I C A / H L A-B$ & 0.4382549 & $2682 / 3892^{\mathrm{a}}$ \\
\hline 6 & rs2474619 & A & 0.66 & 0.64 & 90880035 & $2.75 \times 10^{-3}$ & 1.12 & $\mathrm{BACH} 2$ & 0.1133287 & $2682 / 3892^{a}$ \\
\hline 6 & rs9355610 & $\mathrm{G}$ & 0.51 & 0.48 & 167383075 & $1.34 \times 10^{-4}$ & 1.15 & RNASET2/FGFR1OP & 0.1397619 & $2682 / 3892^{\mathrm{a}}$ \\
\hline 11 & rs 12575636 & $\mathrm{G}$ & 0.11 & 0.08 & 95311260 & $3.59 \times 10^{-5}$ & 1.28 & FAM76B/SESN3 & 0.2468601 & $2682 / 3892^{a}$ \\
\hline 22 & rs229527 & A & 0.75 & 0.72 & 37581485 & $3.00 \times 10^{-6}$ & 1.22 & CIQTNF6 & 0.1988509 & $2682 / 3892^{a}$ \\
\hline $\mathrm{X}$ & rs5912838 & A & 0.64 & 0.58 & 78497118 & $1.58 \times 10^{-10}$ & 1.26 & GPR174/ITM2A & 0.2311117 & $2682 / 3892^{\mathrm{a}}$ \\
\hline
\end{tabular}

The formula for the weighted-GRS is presented as follows: Weighted $-\mathrm{GRS}=\frac{\sum_{i=1}^{n} w_{i} \times X_{i}}{\sum_{i=1}^{n} w_{i}} \times n$, where $i$ is the SNP, $n$ is the number of the
SNPs, W $i=$ weight for SNP $i$, and Xi $i=$ number of risk alleles $(0,1$, or 2$)$. ${ }^{\mathrm{a}}$ Data referenced from Zhang et al. (30).

${ }^{\mathrm{b}}$ Unpublished data.

Chr, chromosome; OR, odds ratio; RAF, risk allele frequency; SNP, single nucleotide polymorphism; weighted-GRS, weighted genetic risk score. 
of the genetic association within each allele. The ORs from the previous large data set and the ORs calculated from our current data set were comparable, with the effect sizes in the same direction (data not shown). The weighted-GRS was calculated as follows: multiplying the number of risk alleles for each SNP $(0,1,2)$ by the weight ( $\beta$-coefficient) for that SNP and then taking the sum across all SNPs. Next, the value of the weighted score was rescaled by dividing all values by the sum of the weights ( $\beta$-coefficients) and then multiplying by the total number of SNPs. The weights used in the weighted-GRS were calculated as the natural log of the OR with respect to the risk allele. Since there is currently no evidence of epistasis among these susceptibility loci (data not shown), we did not add terms for interaction between the loci in our algorithm. The unweighted-GRS ranged from 5 to 21 , and the weightedGRS ranged from 3.97 to 21.59 , with higher scores indicating a higher genetic predisposition to HT. The full list of the SNPs included in the GRSs and the ORs that were used as weights for the weighted-GRS are provided in Table 1.

\section{Statistical analyses}

The normality of the distribution was examined before the analyses using the Kolmogorov-Smirnov test. Continuous data are presented as median and interquartile range (IQR) or mean \pm standard deviation (SD), and differences between cases and controls were examined using the Wilcoxon rank sum tests or $t$-tests as appropriate (31). Categorical data are presented as number (\%), and chi-square tests were used for intergroup comparisons.

We conducted logistic regression models to assess the main effects of urinary iodine concentration and GRSs on the risk of HT controlling for potential confounders. These potential confounders were chosen based on our univariate analysis shown in Table 2, as well as the findings from the previous literature (4,38-41). First, Model 1 was adjusted for no variables. Then, sex, age, education attainment, BMI, total cholesterol, triglyceride, LDL-C, HDL-C, and urinary creatinine (for urinary iodine) were adjusted in Model 2. Urinary iodine concentration as well as GRSs were categorized into quartiles and analyzed in logistic regression models with the lowest quartile as the reference group. $p$-Values for trend were calculated by entering the urinary iodine or GRSs as a continuous variable into the regression models. We also applied restricted cubic spline regression with four knots located at the 5th, 35th, 65th, and 95th percentiles $(31,42-44)$ of urinary iodine to estimate the possible nonlinear relationship between urinary iodine and HT risk adjusting for potential confounders listed in Model 2. The urinary iodine was analyzed as a continuous variable, and ORs and $95 \%$ confidence intervals [CIs] were calculated using the median urinary iodine concentration as the reference value.

For the gene-iodine interactions, crude and multivariable logistic regression models were applied to estimate the risk of HT per increment of 1 -unit of $\log _{10}$-iodine, stratified according to the quartiles of GRSs. The effects of interactions between the GRSs and urinary iodine on the risk of HT were assessed by including the respective interaction terms in the models (e.g., urinary iodine $\times$ weighted-GRS quartiles).

Finally, we fitted multinomial logistic regression models to evaluate the association of urinary iodine with euthyroid-HT and hypothyroidism-HT in both Model 1 and Model 2, using the control as the reference group.

Table 2. Characteristics of the Study Population $(N=1723)$

\begin{tabular}{|c|c|c|c|c|}
\hline Characteristic & Total & Case & Control & $\mathrm{p}$ \\
\hline Samples $(n)$ & 1723 & 731 & 992 & - \\
\hline Age (years) & $43.20 \pm 13.19$ & $42.12 \pm 13.89$ & $44.00 \pm 12.60$ & 0.004 \\
\hline Sex (male/female) & $186 / 1537$ & $46 / 685$ & $140 / 852$ & $<0.001$ \\
\hline $\operatorname{BMI}\left(\mathrm{kg} / \mathrm{m}^{2}\right)$ & $22.95 \pm 3.04$ & $23.35 \pm 3.27$ & $22.66 \pm 2.83$ & $<0.001$ \\
\hline Education attainment (years) & & & & $<0.001$ \\
\hline$\leq 9$ & $439(25.50)$ & $299(40.90)$ & $140(14.10)$ & \\
\hline $9.1-12$ & $318(18.50)$ & $146(20.00)$ & $172(17.30)$ & \\
\hline $12.1-15$ & $444(25.80)$ & $133(18.20)$ & $311(31.40)$ & \\
\hline$\geq 15$ & $522(30.30)$ & $153(20.90)$ & $369(37.20)$ & \\
\hline HDL-C (mM) & $1.54(1.28-1.86)$ & $1.53(1.26-1.86)$ & $1.54(1.29-1.86)$ & 0.232 \\
\hline LDL-C (mM) & $3.39(2.80-4.09)$ & $3.26(2.59-3.99)$ & $3.46(2.91-4.17)$ & $<0.001$ \\
\hline Triglyceride (mM) & $1.33(0.97-1.95)$ & $1.49(1.05-2.27)$ & $1.26(0.93-1.81)$ & $<0.001$ \\
\hline Total cholesterol (mM) & $5.28(4.54-6.12)$ & $5.11(4.32-6.05)$ & $5.36(4.67-6.15)$ & $<0.001$ \\
\hline fT3 $(\mathrm{pg} / \mathrm{mL})$ & $3.31(2.97-3.63)$ & $2.94(2.55-3.33)$ & $3.45(3.21-3.72)$ & $<0.001$ \\
\hline fT4 (ng/dL) & $0.81(0.73-0.90)$ & $0.80(0.66-0.94)$ & $0.82(0.76-0.89)$ & 0.003 \\
\hline $\mathrm{TSH}(\mu \mathrm{IU} / \mathrm{mL})$ & $2.56(1.69-5.15)$ & $6.98(2.92-18.00)$ & $2.10(1.48-2.82)$ & $<0.001$ \\
\hline TGAb (IU/mL) & $13.59(10.00-323.90)$ & $393.20(179.40-786.20)$ & $10.00(10.00-10.94)$ & $<0.001$ \\
\hline TPOAb (IU/mL) & $1.90(0.70-166.50)$ & $288.50(48.50-952.40)$ & $0.90(0.50-1.50)$ & $<0.001$ \\
\hline Urinary creatinine $(\mathrm{g} / \mathrm{L})$ & $0.63(0.29-1.15)$ & $0.68(0.35-1.20)$ & $0.57(0.27-1.13)$ & 0.001 \\
\hline Urinary iodine $(\mu \mathrm{g} / \mathrm{L})$ & $106.70(54.30-191.10)$ & $125.20(65.50-228.30)$ & $95.20(48.63-168.50)$ & $<0.001$ \\
\hline $\begin{array}{l}\text { Urinary iodine-to-creatinine } \\
(\mu \mathrm{g} / \mathrm{g} \text { creatinine })\end{array}$ & $185.05(127.08-287.43)$ & $206.33(135.74-310.95)$ & $172.31(122.22-265.27)$ & $<0.001$ \\
\hline Unweighted-GRS & $12.84 \pm 2.31$ & $13.37 \pm 2.25$ & $12.45 \pm 2.28$ & $9.42 \times 10^{-17}$ \\
\hline Weighted-GRS & $12.48 \pm 2.34$ & $13.04 \pm 2.23$ & $12.06 \pm 2.33$ & $4.03 \times 10^{-18}$ \\
\hline
\end{tabular}

Data are presented as mean \pm standard deviation or median (IQR) for continuous variables and $n(\%)$ for categorical variables.

BMI, body mass index; fT3, free triiodothyronine; fT4, free thyroxine; HDL-C, high-density lipoprotein cholesterol; IQR, interquartile range; LDL-C, low-density lipoprotein cholesterol; TGAb, thyroglobulin antibody; TPOAb, thyroid peroxidase antibody; TSH, thyrotropin. 
Statistical analyses were performed with the use of PLINK (version 1.9; Boston, MA) and SAS (version 9.4; SAS Institute, Inc., Cary, NC). The spline curve was constructed using Stata MP (version 14.2; StataCorp LP, TX) with the "mkspline" command. Statistical significance was defined as a two-tailed $p$-value $<0.05$.

\section{Results}

\section{Characteristics of the study population}

Table 2 presents the characteristics of the study population. Compared with controls, HT patients were more likely to be younger, female, had higher levels of BMI, triglyceride, TPOAb, TGAb, TSH, urinary creatinine, and lower levels of education, fT3, fT4, LDL-C, and total cholesterol (all $p<0.05$ ). The mean (SD) of the unweighted-GRS was 12.84 (2.31) risk alleles, whereas the mean (SD) of the weighted-GRS was 12.48 (2.34) risk alleles for the whole study population. As expected, both the unweighted-GRS and the weighted-GRS were significantly higher among HT patients. The median concentration of urinary iodine and iodine-to-creatinine for the whole study population were $106.70 \mu \mathrm{g} / \mathrm{L} \quad(\mathrm{IQR}=54.30-191.10 \mu \mathrm{g} / \mathrm{L})$ and $185.05 \mu \mathrm{g} / \mathrm{g}$ creatinine $(\mathrm{IQR}=127.08-287.43 \mu \mathrm{g} / \mathrm{g}$ creatinine $)$, respectively, showing that the levels of iodine-to-creatinine were significantly higher than that of urinary iodine $(p<0.001)$. Interestingly, HT participants had higher levels of both urinary iodine $(p<0.001)$ and iodine-to-creatinine $(p<0.001)$ than those without HT.

\section{The effect of urinary iodine and the GRSs on the risk of $H T$}

After adjusting for sex, age, education attainment, BMI, total cholesterol, triglyceride, LDL-C, HDL-C, and urinary creatinine, the odds of HT increased with increasing quartiles of urinary iodine concentration: adjusted ORs and CIs were 1.45 [1.06-1.99], 1.66 [1.17-2.34], and 2.07 [1.38-3.10] for the quartiles 2, 3, and 4, respectively, compared with the first quartile (Model 2, Table 3). Similar results were obtained in Model 1 . With each 1 -unit increase of $\log _{10}$-transformed urine iodine concentration, the odds of HT increased by $139 \%$ (adjusted $\mathrm{OR}=2.39[\mathrm{CI}=1.85-3.08], p$ for trend $<0.001$ ) in Model 1 and 124\% (adjusted $\mathrm{OR}=2.24$ [CI $=1.53-3.28$ ], $p$ for trend $<0.001$ ) in Model 2. Next, to visualize the potential dose-response relationship, the multivariable restricted cubic spline regression was performed predicting ORs for HT between the 5th and 95th percentiles of the population urinary iodine distribution (Fig. 1). Interestingly, the association of urinary iodine with HT risk was near linear $(p$-overall $<0.001$; $p$-nonlinear $=0.074$ ).

As expected, the unweighted-GRS and the weighted-GRS were also significantly related to an increasing risk of HT in both Model 1 (crude OR = 1.20 [CI = 1.15-1.25] for each 1 -point increase of the unweighted-GRS, $p$ for trend $=6.74 \times 10^{-16}$; crude $\mathrm{OR}=1.21[\mathrm{CI}=1.16-1.26]$ for each 1 -point increase of the weighted-GRS, $p$ for trend $=4.09 \times 10^{-17}$ ) and Model 2 (adjusted $\mathrm{OR}=1.19[\mathrm{CI}=1.14-1.25]$ for each 1 -point increase of the unweighted-GRS, $p$ for trend $=8.22 \times 10^{-13}$; adjusted $\mathrm{OR}=1.20[\mathrm{CI}=1.14-1.26]$ for each 1 -point increase of the weighted-GRS, $p$ for trend $=1.40 \times 10^{-13}$ ).

\section{Interaction of urinary iodine concentration and GRSs on HT risk}

As shown in Figure 2, we did not find a significant interaction between urinary iodine concentration and weightedGRS on the risk of HT either in the unadjusted or in the multivariate-adjusted models (all $p$ for interaction $>0.05$ ), with similar results observed for the unweighted-GRS. Specifically, the magnitude of the associations between urinary iodine concentration and HT risk did not change by unweighted-GRS or weighted-GRS quartiles, indicating that GRSs did not modify the association between urinary iodine and HT risk.

\section{The effect of urinary iodine concentration on HT with or without hypothyroidism}

All HT cases were further classified into two groups as "euthyroid-HT" or "hypothyroidism-HT' according to their thyroid function status. As shown in Table 4, when using the control group as the reference category, multinomial logistic regression models demonstrated that each 1 -unit increase of $\log _{10}$-transformed urine iodine concentration was associated with a more than twofold increase in the odds of hypothyroidism-HT even after controlling for sex, age, education attainment, BMI, total cholesterol, triglyceride, LDL-C, HDL-C, and urinary creatinine (adjusted $\mathrm{OR}=2.64[\mathrm{CI}=1.73-4.05]$ ) but was not associated with the risk of euthyroid-HT in both the unadjusted and adjusted analysis (all $p>0.05$ ).

\section{Discussion}

In the present study, an increase in urinary iodine concentration was significantly associated with increased risk of HT even after adjusting for sex, age, education attainment, BMI, total cholesterol, triglyceride, LDL-C, HDL-C, and urinary creatinine. Moreover, this association was near linear, indicating that increased urinary iodine has a continuous and graded impact on HT risk. To the best of our knowledge, this is the first study focusing on the interaction between the iodine concentration and genetic predisposition to HT risk utilizing the Asian-specific HT risk-related GRSs as the modifying factors, which showed no modification effect of the GRSs on the association between urinary iodine and HT. However, when further stratifying HT patients according to their thyroid function status, we found that each increment of urinary iodine concentration was associated with a more than twofold increase in the odds of hypothyroidism-HT, but not with euthyroid-HT, suggesting the possibility that increased iodine might accelerate the development of hypothyroidism rather than trigger the presence of thyroid autoantibodies in normal population, which still needs to be explored further in future longitudinal studies.

Although the prevalence of HT, as the most common autoimmune thyroiditis, is still increasing rapidly $(4,8)$, the data regarding the effect of urinary iodine on HT are rather inconsistent (9-16). For example, in a cross-sectional study comprising 764 young women with goiters who went through fine-needle aspiration cytology successfully (9), Marwaha et al. did not find any relationship of urinary iodine with juvenile autoimmune thyroiditis as diagnosed by the presence of cytopathologic features consistent with either HT or focal 


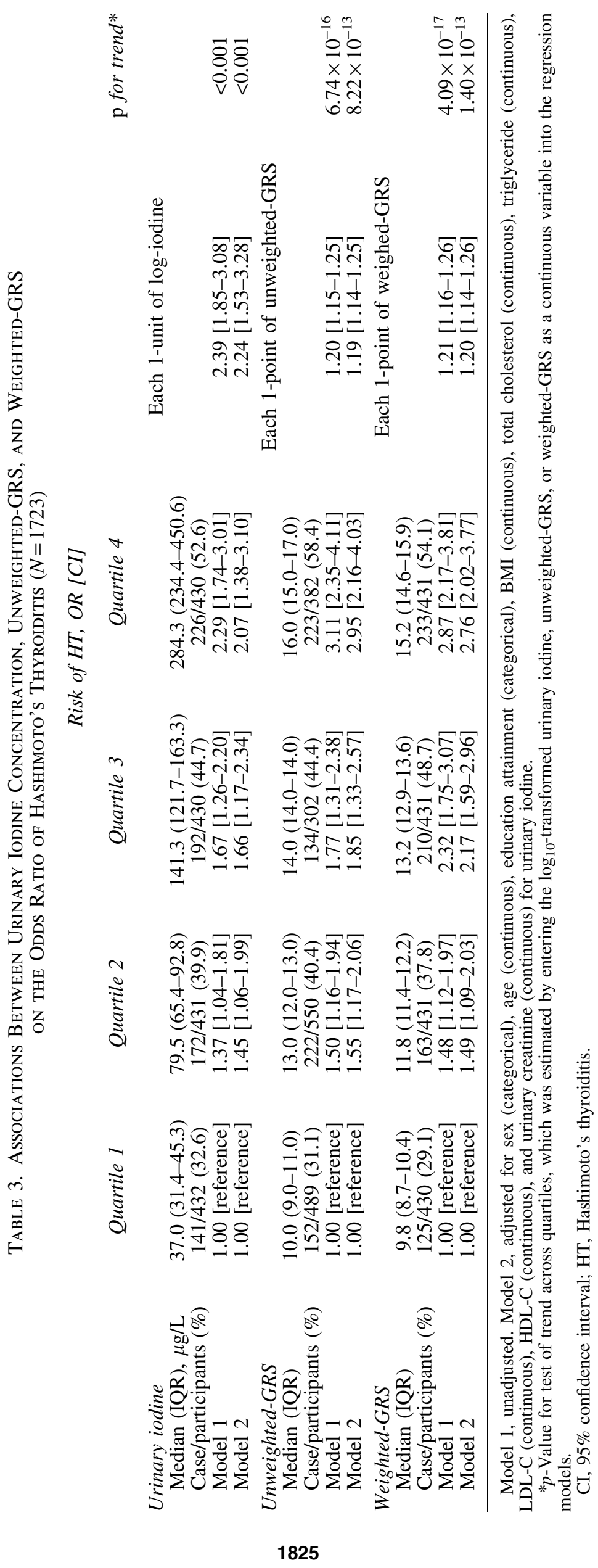




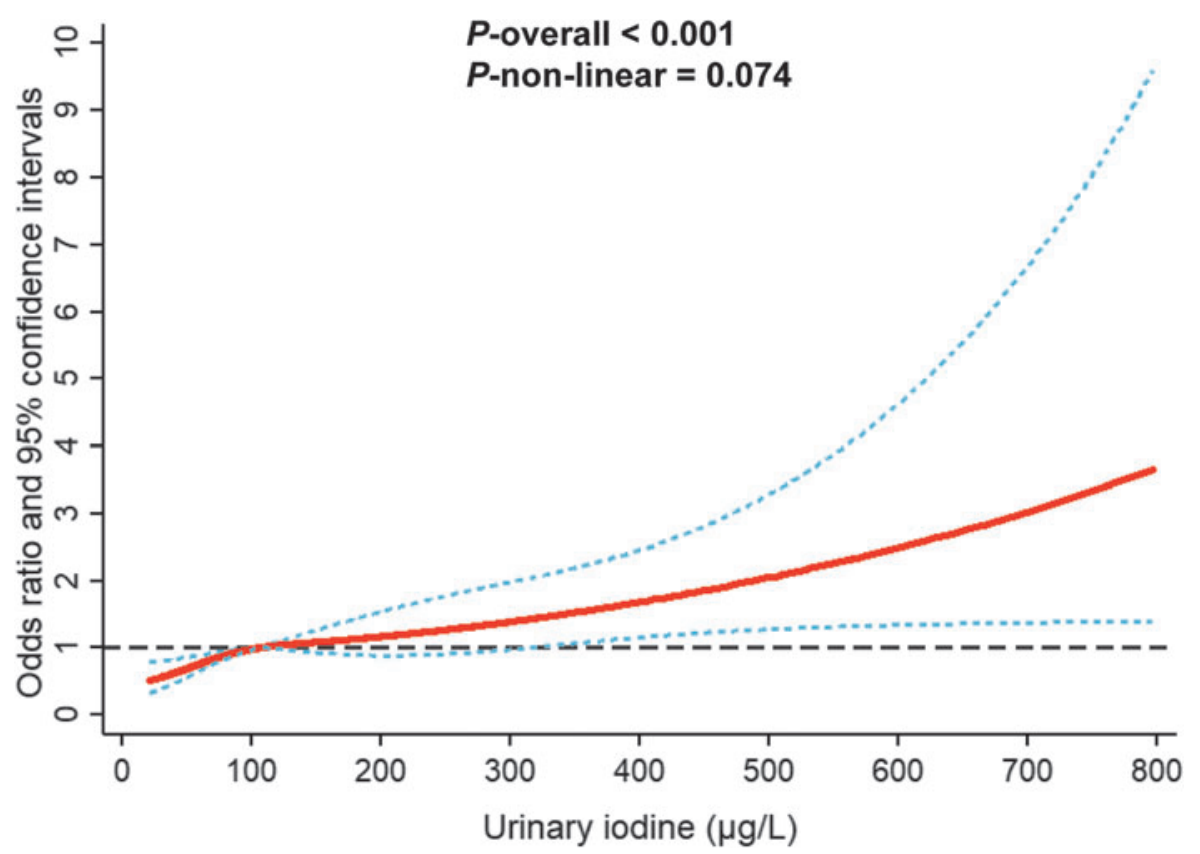

FIG. 1. Association between urinary iodine and HT risk. The restricted cubic spline regression model with four knots located at the 5th, 35th, 65th, and 95th percentiles of urinary iodine $(30.5,72.7,145.0$, and 403.8 $\mu \mathrm{g} / \mathrm{L})$. ORs (red solid line) and CIs (blue dashed lines) were estimated using the median urinary iodine concentration as the reference value $(106.7 \mu \mathrm{g} / \mathrm{L})$. The horizontal dashed line represents the reference OR of 1.0. The model was adjusted for sex (categorical), age (continuous), education attainment (categorical), BMI (continuous), total cholesterol (continuous), triglyceride (continuous), LDL-C (continuous), HDL-C (continuous), and urinary creatinine (continuous). BMI, body mass index; CI, 95\% confidence interval; HDL-C, high-density lipoprotein cholesterol; HT, Hashimoto's thyroiditis; LDL-C, low-density lipoprotein cholesterol; OR, odds ratio.

lymphocytic thyroiditis. However, the cross-sectional study of Wang et al. showed an U-shaped relationship of iodine intake with HT in adults (11), whereas Chen et al. reported that iodine deficiency, other than excessive and more than adequate iodine nutrition, was associated with increased risk of HT (12), both of which defined HT only by the presence of positive TGAb or TPOAb. By contrast, we reported that urinary iodine concentration was associated with increased risk of HT as diagnosed by the presence of diffuse swelling of the thyroid gland, the positivity for TPOAb or TGAb, and hypoechogenicity of the thyroid parenchyma on ultrasound, which is consistent with the population-based studies of Shan et al. (13), Teng et al. (14), and Aghini Lombardi et al. (15) who compared data in regions with different iodine intake, as well as the experimental data from animal models $(1,20)$, corroborating that the prevalence of HT significantly increased with an increasing iodine intake. Furthermore, with the use of restricted cubic spline regression analysis estimated by entering the urinary iodine as a continuous variable, we observed that the relationship between urinary iodine concentration and HT risk was near linear. Such heterogeneous results might be attributed, at least partly, to the differences in urinary iodine concentration or the criteria adopted for HT diagnosis. The median urinary iodine concentration of the population in the current study was $106.7 \mu \mathrm{g} / \mathrm{L}$, showing an adequate iodine intake (median urinary iodine concentration,
FIG. 2. OR of HT per increment of 1-unit of logiodine according to the quartile of the genetic risk scores. Model 1, unadjusted. Model 2, adjusted for sex (categorical), age (continuous), education attainment (categorical), BMI (continuous), total cholesterol (continuous), triglyceride (continuous), LDL-C (continuous), HDL-C (continuous), and urinary creatinine (continuous). Horizontal bars indicate CIs.

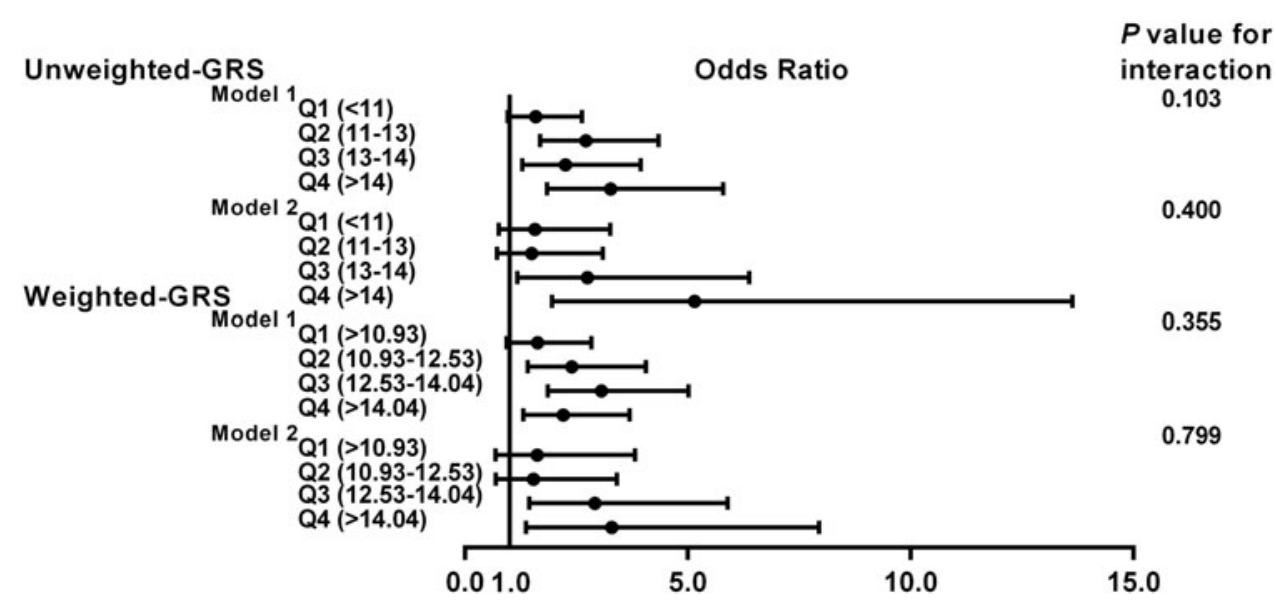


Table 4. Multinomial Logistic Regression Analyses of Urinary Iodine with Hashimoto's Thyroiditis Status

\begin{tabular}{llcc}
\hline & \multicolumn{3}{c}{ OR $[$ CI] } \\
\cline { 2 - 4 } & Control $(\mathrm{n}=992)$ & Euthyroid-HT $(\mathrm{n}=220)$ & Hypothyroidism-HT $(\mathrm{n}=511)$ \\
\hline Model 1 & 1.00 [reference] & $1.18[0.80-1.74]$ & $3.23[2.42-4.30]^{*}$ \\
Model 2 & 1.00 [reference] & $1.64[0.95-2.81]$ & $2.64[1.73-4.05]^{*}$ \\
\hline
\end{tabular}

Model 1, unadjusted. Model 2, adjusted for sex (categorical), age (continuous), education attainment (categorical), BMI (continuous), total cholesterol (continuous), triglyceride (continuous), LDL-C (continuous), HDL-C (continuous), and urinary creatinine (continuous). $* p<0.05$.

$100-199 \mu \mathrm{g} / \mathrm{L})$. Nevertheless, it is worth mentioning that the proportion of iodine deficiency (defined as the median urinary iodine concentration $<100 \mu \mathrm{g} / \mathrm{L}$ ) was $46.4 \%$. The level of urinary iodine was lower, and the proportion of iodine deficiency was higher in our study than that of the majority of studies mentioned above except for Chen et al. [e.g., in the study of Shan et al. (13), the median urinary iodine concentration was $205 \mu \mathrm{g} / \mathrm{L}$; the proportion of iodine deficiency was only $12.63 \%$ ]. After implementation of universal salt iodization programs to prevent iodine deficiency disorders, China has been moved from a condition of chronic iodine deficiency to more than adequate. Since the participants with iodine deficiency in the current study were more likely to have a higher educational attainment than those without (the percentage of more than 12 years of education: $62.0 \%$ versus $50.9 \%$, $p<0.001$ ), it is possible that individuals with a higher academic background who have easy access to the media or medical literature may conceivably be more preferable for consuming non-iodized salt, thereby reflecting a lower value of urinary iodine. Interestingly, our data of urinary iodine concentration were highly consistent with the survey recently reported by Chen et al. (12), which showed the median urinary iodine concentration of $106.4 \mu \mathrm{g} / \mathrm{L}$ and the iodine deficiency proportion of $46.8 \%$ in urban dwellers in downtown Shanghai. Most importantly, by stratifying the HT cases according to their thyroid function, we found that each increment of urinary iodine was significantly associated with increased risk of hypothyroidism-HT, but not associated with the risk of euthyroid-HT, raising the hypothesis that instead of triggering autoimmune dysfunction, increased urinary iodine might drive thyroid function from a state of potential autoimmune impairment toward hypothyroidism. Indeed, this is supported by a follow-up study conducted among 213 subjects with euthyroidism who had high levels of either TPOAb or TGAb at baseline, which showed that the five-year incidence of hypothyroidism was greater among subjects with more than adequate or excessive iodine intake than those with mildly deficient iodine intake (14).

Potential biological mechanisms that might explain the association between urinary iodine concentration and increased risk of HT-associated hypothyroidism is unclear. Valuable evidence from experimental studies suggest that iodine exposure may cause a higher generation of radical oxygen species (ROS) in the thyrocyte during TPO oxidation of excessive amounts of iodine, thereby activating pathways promoting thyrocyte destruction $(4,45-48)$. In addition, since apoptosis has been described as an important pathway contributing to the thyrocytes destruction in HT $(4,49,50)$, and was also induced following exposure to iodine $(47,51,52)$, the effect of iodine on apoptosis might be potential mechanism. Finally, it is also worth noting that high iodine intake reduces the synthesis of the thyroid hormones through the acute Wolff-Chaikoff effect (53). Interestingly, certain susceptible individuals with underlying thyroid disease (frequently autoimmune thyroid disease) may fail to adapt to or escape from the Wolff-Chaikoff effect caused by the inability of downregulation of the sodium-iodine symporter $(54,55)$, thereby leading to the development of hypothyroidism when exposed to high levels of iodine. This may explain, at least partly, why higher urinary iodine levels were associated with increased risks of hypothyroid-HT, but not in controls (as observed in our study).

Another interesting result of the current study is that we did not detect a significant interaction between urinary iodine concentration and the genetic predisposition to HT risk, either using the weighted or unweighted GRS showing consistent results. It seems that HT-related genetic variations cannot modify the effect of increased iodine concentration on HT risk. Considering the fact that more than half of these selected HT loci harbor genes known to be related to immune function, it is possible to imply that non-immune-based mechanisms might underlie or contribute to the iodine-HT association. Indeed, it is supported by our finding that urinary iodine was not significantly associated with increased risk of euthyroid-HT, implying that increased iodine might not induce the autoimmune disturbance in healthy individuals. However, because the biological functions of most HT loci remain largely unexplained, the exact mechanisms underlying the identified observation need to be clarified in future studies.

Several limitations need to be acknowledged. First, the use of urinary iodine concentration $(\mu \mathrm{g} / \mathrm{L})$ to estimate iodine status from single spot urine sample is an important limitation. The most precise method of estimating population iodine intake is the measurement of 24-hour urinary iodine excretion $(\mu \mathrm{g} /$ day $)(33,34)$, which for the most part can reflect an individual's long-term iodine status. However, collecting 24-hour urine samples harbors the risk of lower compliance, which may compromise data quality especially in field studies (33). Urinary iodine excretion can vary somewhat from day-to-day and even within a given day $(33,34,56)$, limiting the use of spot urine samples as an approach for monitoring iodine status. However, it is important to note that this variation usually tends to even out among populations (34). Indeed, the WHO currently recommends using the urine iodine concentration $(\mu \mathrm{g} / \mathrm{L})$ from spot urine sample to evaluate the iodine status of a population, whereas it describes the use of iodine-to-creatinine ( $\mu \mathrm{g} / \mathrm{g}$ creatinine) as "unreliable", 
and "unnecessary" (36) since creatinine excretion varies by $\mathrm{pH}$ condition, protein intake, muscle mass, ethnic background, age, and sex (35). In fact, we confirmed in the current study that although iodine-to-creatinine correlated with urinary iodine concentration $(r=0.16, p<0.001)$, the level of iodine-to-creatinine was significantly higher than that of urinary iodine $(p<0.001)$. When iodine-to-creatinine was further analyzed in the regression models using the same statistical methods as urinary iodine concentration, it demonstrated that the results of iodine-to-creatinine were in agreement with that of urinary iodine in our study (data not shown). A further limitation is that our GRSs captured the combined information from the 13 established HT associated loci that have been identified in Asians to date (30), but it contains only a small amount of variation in HT. Further genetic studies that might account for a significantly larger proportion of HT heritability are needed. Our study was restricted to the population of Asian ancestry; therefore, caution is needed if generalizing the findings to other ethnic groups. In addition, despite several most likely confounders adjusted for in the multivariable regression including sex, age, education attainment, BMI, total cholesterol, triglyceride, LDL-C, HDL-C, and urinary creatinine, some residual factors may not be fully ruled out at this time. Finally, future prospective studies determining a causal relationship are highly warranted.

In conclusion, our results indicated that an increase in urinary iodine concentration was associated with increased risk of $\mathrm{HT}$, and the association is not modified by genetic predisposition to HT. Moreover, urinary iodine concentration was significantly associated with increased risk of hypothyroidismHT. These findings suggest that currently there is no evidence to support for personalized advice on iodine intake for HT prevention in individuals with different levels of genetic risk for HT.

\section{Acknowledgments}

We thank all the involved technicians, nurses, and clinicians for their skill, time and commitment to this study.

\section{Author Disclosure Statement}

No competing financial interests exist.

\section{Funding Information}

This work was supported by the Shanghai Sailing Program of Shanghai Science and Technology Commission (20YF14 23900), the programs of the National Key R\&D Program of China (2017YFC1001801), and the National Natural Science Foundation of China (81661168016, 81870540, 81770786, 81800749, and 81430019).

\section{References}

1. Burek CL, Talor MV 2009 Environmental triggers of autoimmune thyroiditis. J Autoimmun 33:183-189.

2. Vanderpump MP 2011 The epidemiology of thyroid disease. Br Med Bull 99:39-51.

3. Biondi B, Cooper DS 2008 The clinical significance of subclinical thyroid dysfunction. Endocr Rev 29:76-131.

4. Zaletel K, Gaberscek S 2011 Hashimoto's thyroiditis: from genes to the disease. Curr Genomics 12:576-588.
5. Caturegli P, De Remigis A, Rose NR 2014 Hashimoto thyroiditis: clinical and diagnostic criteria. Autoimmun Rev 13:391-397.

6. Versini M, Jeandel PY, Rosenthal E, Shoenfeld Y 2014 Obesity in autoimmune diseases: not a passive bystander. Autoimmun Rev 13:981-1000.

7. McLeod DS, Cooper DS 2012 The incidence and prevalence of thyroid autoimmunity. Endocrine 42:252-265.

8. Rizzo M, Rossi RT, Bonaffini O, Scisca C, Altavilla G, Calbo L, Rosano A, Sindoni A, Trimarchi F, Benvenga S 2010 Increased annual frequency of Hashimoto's thyroiditis between years 1988 and 2007 at a cytological unit of Sicily. Ann Endocrinol (Paris) 71:525-534.

9. Marwaha RK, Tandon N, Karak AK, Gupta N, Verma K, Kochupillai N 2000 Hashimoto's thyroiditis: countrywide screening of goitrous healthy young girls in postiodization phase in India. J Clin Endocrinol Metab 85: 3798-3802.

10. Miranda DM, Massom JN, Catarino RM, Santos RT, Toyoda SS, Marone MM, Tomimori EK, Monte O 2015 Impact of nutritional iodine optimization on rates of thyroid hypoechogenicity and autoimmune thyroiditis: a crosssectional, comparative study. Thyroid 25:118-124.

11. Wang B, He W, Li Q, Jia X, Yao Q, Song R, Qin Q, Zhang JA 2019 U-shaped relationship between iodine status and thyroid autoimmunity risk in adults. Eur J Endocrinol 181: 255-266.

12. Chen $\mathrm{C}, \mathrm{Xu} \mathrm{H}$, Chen Y, Chen Y, Li Q, Hu J, Liang W, Cheng J, Xia F, Wang C, Han B, Zheng Y, Jiang B, Wang N, Lu Y 2017 Iodized salt intake and its association with urinary iodine, thyroid peroxidase antibodies, and thyroglobulin antibodies among urban Chinese. Thyroid 27: 1566-1573.

13. Shan Z, Chen L, Lian X, Liu C, Shi B, Shi L, Tong N, Wang S, Weng J, Zhao J, Teng X, Yu X, Lai Y, Wang W, Li C, Mao J, Li Y, Fan C, Teng W 2016 Iodine status and prevalence of thyroid disorders after introduction of mandatory universal salt iodization for 16 years in China: a cross-sectional study in 10 cities. Thyroid 26:11251130 .

14. Teng W, Shan Z, Teng X, Guan H, Li Y, Teng D, Jin Y, Yu X, Fan C, Chong W, Yang F, Dai H, Yu Y, Li J, Chen Y, Zhao D, Shi X, Hu F, Mao J, Gu X, Yang R, Tong Y, Wang W, Gao T, Li C 2006 Effect of iodine intake on thyroid diseases in China. N Engl J Med 354:2783-2793.

15. Aghini Lombardi F, Fiore E, Tonacchera M, Antonangeli L, Rago T, Frigeri M, Provenzale AM, Montanelli L, Grasso L, Pinchera A, Vitti P 2013 The effect of voluntary iodine prophylaxis in a small rural community: the Pescopagano survey 15 years later. J Clin Endocrinol Metab 98: 1031-1039.

16. Petersen M, Knudsen N, Carle A, Andersen S, Jorgensen T, Perrild H, Ovesen L, Rasmussen LB, Thuesen BH, Pedersen IB 2019 Increased incidence rate of hypothyroidism after iodine fortification in Denmark: a 20-year prospective population-based study. J Clin Endocrinol Metab 104: 1833-1840.

17. Markou K, Georgopoulos N, Kyriazopoulou V, Vagenakis AG 2001 Iodine-Induced hypothyroidism. Thyroid 11:501510.

18. Braverman LE, Ingbar SH, Vagenakis AG, Adams L, Maloof F 1971 Enhanced susceptibility to iodide myxedema in patients with Hashimoto's disease. J Clin Endocrinol Metab 32:515-521. 
19. Reinhardt W, Luster M, Rudorff KH, Heckmann C, Petrasch S, Lederbogen S, Haase R, Saller B, Reiners C, Reinwein D, Mann K 1998 Effect of small doses of iodine on thyroid function in patients with Hashimoto's thyroiditis residing in an area of mild iodine deficiency. Eur $\mathbf{J}$ Endocrinol 139:23-28.

20. Teng X, Shan Z, Teng W, Fan C, Wang H, Guo R 2009 Experimental study on the effects of chronic iodine excess on thyroid function, structure, and autoimmunity in autoimmuneprone NOD.H-2h4 mice. Clin Exp Med 9:51-59.

21. Li HS, Carayanniotis G 2007 Induction of goitrous hypothyroidism by dietary iodide in SJL mice. Endocrinology 148:2747-2752.

22. Brix TH, Hegedus L 2012 Twin studies as a model for exploring the aetiology of autoimmune thyroid disease. Clin Endocrinol (Oxf) 76:457-464.

23. Brcic L, Baric A, Gracan S, Brdar D, Torlak Lovric V, Vidan N, Zemunik T, Polasek O, Barbalic M, Punda A, Boraska Perica V 2016 Association of established thyroid peroxidase autoantibody (TPOAb) genetic variants with Hashimoto's thyroiditis. Autoimmunity 49:480485.

24. Zaaber I, Mestiri S, Marmouch H, Mahjoub S, Abid N, Hassine M, Bel Hadj Jrad-Tensaout B, Said K 2014 Polymorphisms in TSHR and IL1RN genes and the risk and prognosis of Hashimoto's thyroiditis. Autoimmunity 47: 113-118.

25. Yan N, Yu YL, Yang J, Qin Q, Zhu YF, Wang X, Song RH, Zhang JA 2012 Association of interleukin-17A and -17F gene single-nucleotide polymorphisms with autoimmune thyroid diseases. Autoimmunity 45:533-539.

26. Santos LR, Duraes C, Mendes A, Prazeres H, Alvelos MI, Moreira CS, Canedo P, Esteves C, Neves C, Carvalho D, Sobrinho-Simoes M, Soares P 2014 A polymorphism in the promoter region of the selenoprotein $\mathrm{S}$ gene (SEPS1) contributes to Hashimoto's thyroiditis susceptibility. J Clin Endocrinol Metab 99:E719-E723.

27. Ajjan RA, Weetman AP 2015 The Pathogenesis of Hashimoto's Thyroiditis: further Developments in our Understanding. Horm Metab Res 47:702-710.

28. Brcic L, Baric A, Gracan S, Brekalo M, Kalicanin D, Gunjaca I, Torlak Lovric V, Tokic S, Radman M, Skrabic V, Miljkovic A, Kolcic I, Stefanic M, Glavas-Obrovac L, Lessel D, Polasek O, Zemunik T, Barbalic M, Punda A, Boraska Perica V 2019 Genome-wide association analysis suggests novel loci for Hashimoto's thyroiditis. J Endocrinol Invest 42:567-576.

29. Jabrocka-Hybel A, Skalniak A, Piatkowski J, TurekJabrocka R, Vyhouskaya P, Ludwig-Slomczynska A, Machlowska J, Kapusta P, Malecki M, Pach D, TrofimiukMuldner M, Lizis-Kolus K, Hubalewska-Dydejczyk A 2018 How much of the predisposition to Hashimoto's thyroiditis can be explained based on previously reported associations? J Endocrinol Invest 41:1409-1416.

30. Zhang QY, Liu W, Li L, Du WH, Zuo CL, Ye XP, Zhou Z, Yuan FF, Ma YR, Sun F, Yu SS, Xie HJ, Zhang CR, Ying YX, Yuan GY, Gao GQ, Liang J, Zhao SX, Song HD 2020 Genetic study in a large cohort supported different pathogenesis of Graves' diseases and Hashimoto's hypothyroidism. J Clin Endocrinol Metab 105:e2600-e2608.

31. Li L, Ying Y, Zhang C, Wang W, Li Y, Feng Y, Liang J, Song H, Wang Y 2019 Bisphenol A exposure and risk of thyroid nodules in Chinese women: a case-control study. Environ Int 126:321-328.
32. Dunn JT, Crutchfield HE, Gutekunst R, Dunn AD 1993 Two simple methods for measuring iodine in urine. Thyroid 3:119-123.

33. Vejbjerg P, Knudsen N, Perrild H, Laurberg P, Andersen S, Rasmussen LB, Ovesen L, Jorgensen T 2009 Estimation of iodine intake from various urinary iodine measurements in population studies. Thyroid 19:1281-1286.

34. Zimmermann MB, Andersson M 2012 Assessment of iodine nutrition in populations: past, present, and future. Nutr Rev 70:553-570.

35. Barr DB, Wilder LC, Caudill SP, Gonzalez AJ, Needham LL, Pirkle JL 2005 Urinary creatinine concentrations in the U.S. population: implications for urinary biologic monitoring measurements. Environ Health Perspect 113:192200.

36. WHO/UNICEF/ICCIDD 2007 Assessment of Iodine Deficiency Disorders and Monitoring Their Elimination: A Guide for Program Managers. Third edition. World Health Organization, Geneva.

37. Chu X, Pan CM, Zhao SX, Liang J, Gao GQ, Zhang XM, Yuan GY, Li CG, Xue LQ, Shen M, Liu W, Xie F, Yang SY, Wang HF, Shi JY, Sun WW, Du WH, Zuo CL, Shi JX, Liu BL, Guo CC, Zhan M, Gu ZH, Zhang XN, Sun F, Wang ZQ, Song ZY, Zou CY, Sun WH, Guo T, Cao HM, Ma JH, Han B, Li P, Jiang H, Huang QH, Liang L, Liu LB, Chen G, Su Q, Peng YD, Zhao JJ, Ning G, Chen Z, Chen JL, Chen SJ, Huang W, Song HD 2011 A genome-wide association study identifies two new risk loci for Graves' disease. Nat Genet 43:897-901.

38. Shukla SK, Singh G, Ahmad S, Pant P 2018 Infections, genetic and environmental factors in pathogenesis of autoimmune thyroid diseases. Microb Pathog 116:279-288.

39. Ong KK, Kuh D, Pierce M, Franklyn JA 2013 Childhood weight gain and thyroid autoimmunity at age 60-64 years: the 1946 British birth cohort study. J Clin Endocrinol Metab 98: 1435-1442.

40. Oh HS, Kwon H, Ahn J, Song E, Park S, Kim M, Han M, Jeon MJ, Kim WG, Kim WB, Shong YK, Rhee EJ, Kim TY 2018 Association between thyroid dysfunction and lipid profiles differs according to age and sex: results from the Korean National Health and Nutrition Examination Survey. Thyroid 28:849-856.

41. Biyikli HH, Arduc A, Isik S, Ozuguz U, Caner S, Dogru F, Shorbagi AI, Erden G, Berker D, Guler S 2014 Assessing the relationship between serum ghrelin levels and metabolic parameters and autoimmunity in patients with euthyroid Hashimoto's thyroiditis. Endocr Pract 20:818-824.

42. Desquilbet L, Mariotti F 2010 Dose-response analyses using restricted cubic spline functions in public health research. Stat Med 29:1037-1057.

43. Greenland S 1995 Avoiding power loss associated with categorization and ordinal scores in dose-response and trend analysis. Epidemiology 6:450-454.

44. Inoue K, Leung AM, Sugiyama T, Tsujimoto T, Makita N, Nangaku M, Ritz BR 2018 Urinary iodine concentration and mortality among U.S. adults. Thyroid 28:913920.

45. Sharma R, Traore K, Trush MA, Rose NR, Burek CL 2008 Intracellular adhesion molecule-1 up-regulation on thyrocytes by iodine of non-obese diabetic.H2(h4) mice is reactive oxygen species-dependent. Clin Exp Immunol 152: 13-20.

46. Vitale M, Di Matola T, D’Ascoli F, Salzano S, Bogazzi F, Fenzi G, Martino E, Rossi G 2000 Iodide excess induces 
apoptosis in thyroid cells through a p53-independent mechanism involving oxidative stress. Endocrinology 141: 598-605.

47. Kolypetri P, Carayanniotis G 2014 Apoptosis of NOD.H2 h4 thyrocytes by low concentrations of iodide is associated with impaired control of oxidative stress. Thyroid 24:11701178 .

48. Weetman AP 2004 Cellular immune responses in autoimmune thyroid disease. Clin Endocrinol (Oxf) 61:405-413.

49. Mitsiades N, Poulaki V, Kotoula V, Mastorakos G, TseleniBalafouta S, Koutras DA, Tsokos M 1998 Fas/Fas ligand up-regulation and Bcl-2 down-regulation may be significant in the pathogenesis of Hashimoto's thyroiditis. J Clin Endocrinol Metab 83:2199-2203.

50. Kaczmarek E, Lacka K, Jarmolowska-Jurczyszyn D, Sidor A, Majewski P 2011 Changes of B and T lymphocytes and selected apopotosis markers in Hashimoto's thyroiditis. J Clin Pathol 64:626-630.

51. Duntas LH 2018 The catalytic role of iodine excess in loss of homeostasis in autoimmune thyroiditis. Curr Opin Endocrinol Diabetes Obes 25:347-352.

52. Yu X, Li L, Li Q, Zang X, Liu Z 2011 TRAIL and DR5 promote thyroid follicular cell apoptosis in iodine excessinduced experimental autoimmune thyroiditis in NOD mice. Biol Trace Elem Res 143:1064-1076.

53. Wolff J, Chaikoff IL 1948 Plasma inorganic iodide as a homeostatic regulator of thyroid function. J Biol Chem 174:555-564.

54. Leung AM, Braverman LE 2014 Consequences of excess iodine. Nat Rev Endocrinol 10:136-142.

55. Leung AM, Braverman LE 2012 Iodine-induced thyroid dysfunction. Curr Opin Endocrinol Diabetes Obes 19:414419.
56. Rasmussen LB, Ovesen L, Christiansen E 1999 Day-to-day and within-day variation in urinary iodine excretion. Eur $\mathbf{J}$ Clin Nutr 53:401-407.

Address correspondence to: Yan Wang, PhD

The Core Laboratory in Medical Center of Clinical Research

Department of Molecular Diagnostics and Endocrinology

Shanghai Ninth People's Hospital

State Key Laboratory of Medical Genomics

Shanghai Jiao Tong University (SJTU)

School of Medicine

Shanghai 200011

China

E-mail: wangyan@shsmu.edu.cn

Huai-Dong Song, MD, PhD

The Core Laboratory in Medical Center

of Clinical Research

Department of Molecular Diagnostics and Endocrinology

Shanghai Ninth People's Hospital State Key Laboratory of Medical Genomics Shanghai Jiao Tong University (SJTU) School of Medicine Shanghai 200011

China

E-mail: huaidong_s1966@163.com 